\title{
Comparison of the Debye-Huckel and the Mean Spherical Approximation Theories for Electrolyte Solutions
}

\author{
Maribo-Mogensen, Bjørn; Kontogeorgis, Georgios M.; Thomsen, Kaj
}

Published in:

Industrial \& Engineering Chemistry Research

Link to article, DOI:

10.1021/ie2029943

Publication date:

2012

Document Version

Publisher's PDF, also known as Version of record

Link back to DTU Orbit

Citation (APA):

Maribo-Mogensen, B., Kontogeorgis, G. M., \& Thomsen, K. (2012). Comparison of the Debye-Huckel and the Mean Spherical Approximation Theories for Electrolyte Solutions. Industrial \& Engineering Chemistry Research, 51(14), 5353-5363. https://doi.org/10.1021/ie2029943

\section{General rights}

Copyright and moral rights for the publications made accessible in the public portal are retained by the authors and/or other copyright owners and it is a condition of accessing publications that users recognise and abide by the legal requirements associated with these rights.

- Users may download and print one copy of any publication from the public portal for the purpose of private study or research.

- You may not further distribute the material or use it for any profit-making activity or commercial gain

- You may freely distribute the URL identifying the publication in the public portal 


\title{
Comparison of the Debye-Hückel and the Mean Spherical Approximation Theories for Electrolyte Solutions
}

\author{
Bjørn Maribo-Mogensen,* Georgios M. Kontogeorgis, and Kaj Thomsen
}

Center for Energy Resources Engineering, Department of Chemical and Biochemical Engineering, Technical University of Denmark (DTU), Søltofts Plads, Building 229, 2800 Kgs. Lyngby, Denmark

\section{Supporting Information}

ABSTRACT: The thermodynamics of electrolyte solutions has been investigated by many scientists throughout the last century. While several theories have been presented, the most popular models for the electrostatic interactions are based on the DebyeHückel and mean spherical approximation (MSA) theories. In this paper we investigate the differences between the DebyeHückel and the MSA theories, and comparisons of the numerical results for the Helmholtz energy and its derivatives with respect to temperature, volume and composition are presented. The investigation shows that the nonrestricted primitive MSA theory performs similarly to Debye-Hückel, despite the differences in the derivation. We furthermore show that the static permittivity is a key parameter for both models and that in many cases it completely dominates the results obtained from the two models. Consequently, we conclude that the simpler Debye-Hückel theory may be used in connection with electrolyte equations of state without loss of accuracy.

\section{INTRODUCTION}

Solutions containing electrolytes are encountered in many important industrial processes, such as postcombustion $\mathrm{CO}_{2}$ capture, acid gas scrubbing, purification of proteins and pharmaceuticals, and corrosion in wet gas pipelines. ${ }^{1}$ To describe the phase equilibrium of mixtures containing electrolytes, it is necessary to account for the long-range electrostatic forces between charged molecules. ${ }^{1}$ The thermodynamics of electrolyte solutions has been studied by many researchers by adding the long-range electrostatic forces to an existing model for the short-range forces in activity coefficient models such as UNIQUAC ${ }^{2}$ and $\mathrm{NRTL}^{3}$ and to equations of state such as SRK or $\mathrm{CPA}^{4-7}$ and a range of SAFT models. ${ }^{8-11}$

The most commonly used models for the electrolyte interactions are the Debye-Hückel model ${ }^{12}$ and the electrostatic part of the mean spherical approximation (MSA). ${ }^{13,14}$ Both models use Coulomb's law to describe the force between two charges $q_{i}$ and $q_{j}$ :

$$
F(r)=\frac{1}{4 \pi} \frac{1}{\varepsilon_{\mathrm{r}} \varepsilon_{0}} \frac{q_{i} q_{j}}{r^{2}}
$$

where $q_{i}$ is the charge of molecule $i, \varepsilon_{\mathrm{r}}$ is the relative static permittivity (or dielectric constant), $\varepsilon_{0}$ is the vacuum permittivity, and $r$ is the distance between the two charges. The Coulombic forces are lowered when the relative static permittivity of the medium increases (e.g., in water with $\varepsilon_{\mathrm{r}}=78$ at $25{ }^{\circ} \mathrm{C}$ ) which enables the dissociation of salts. Another fundamental equation is the electroneutrality condition, that is, that the net charge of a system is zero:

$$
\sum_{i} \rho_{i} q_{i}=0
$$

where $\rho_{i}$ is the density of molecule $i$. The Debye-Hückel model $^{12}$ was first derived by Debye and Hückel in 1923, from a linearization of the Poisson equation and by treating all ions as point charges and assuming a minimum distance of $d_{i}$ between the ions surrounding a central ion. The nonrestricted primitive MSA model was developed by Blum ${ }^{13,14}$ from statistical mechanics, by treating ions as charged hard spheres of different diameters. In the original MSA model, the screening length $(2 \Gamma)^{-1}$ must be solved for numerically as it is done in this work, but several simplifications of the MSA theory making it into an explicit equation of the Helmholtz energy have been presented in literature. ${ }^{1,8}$ Figure 1 shows a visual interpretation of the assumptions of the two models.

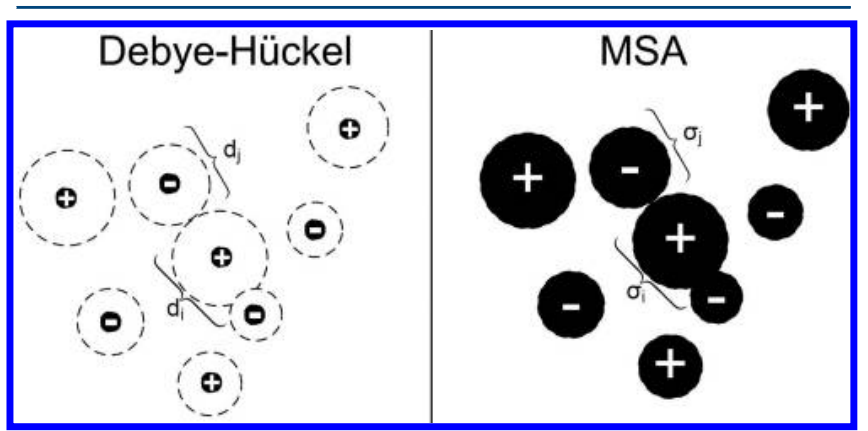

Figure 1. Illustration of the differences between the assumptions of the Debye-Hückel and of the MSA model. Debye-Hückel treats ions as point charges with a minimum separation distance, while MSA treats all ions as charged hard spheres.

Equations of state may be derived from the assumption that the total residual Helmholtz energy may be determined as a sum of the contributions from individual energetic interactions

Received: December 21, 2011

Revised: March 6, 2012

Accepted: March 8, 2012

Published: March 8, 2012 
as shown in eq 3. For example, Inchekel et al. ${ }^{7}$ formed an equation of state (EoS) based on the cubic plus association (CPA) model $^{1}$ where the short-range forces were calculated using SRK, ${ }^{15}$ the effect of hydrogen bonding was calculated by the association theory from SAFT, ${ }^{16}$ the effect of ion solvation using a Born term, ${ }^{17}$ and the long-range electrostatic forces were modeled with MSA: ${ }^{13,14}$

$$
A^{\mathrm{r}}=A^{\mathrm{SRK}}+A^{\text {association }}+A^{\mathrm{MSA}}+A^{\text {Born }}
$$

The Helmholtz energy depends on temperature, volume, and composition, while normally we specify temperature, pressure, and composition. The pressure may be calculated using

$$
P=-R T\left(\frac{\partial}{\partial V}\left(\frac{A^{\mathrm{r}}}{R T}\right)-\frac{n_{\mathrm{T}}}{V}\right)
$$

So to match the specification of temperature and pressure, an EoS must solve the pressure equation given by eq 4 . As the pressure is a function of all terms in eq 3 , the volume becomes a function of the volume derivative of all models in eq 3 . To calculate the equilibrium at a given temperature, pressure, and composition, we need to calculate the fugacity coefficients using

$$
\ln \hat{\varphi}_{i}=\frac{\partial}{\partial n_{i}}\left(\frac{A^{\mathrm{r}}}{R T}\right)-\ln Z
$$

where $Z$ is the compressibility factor. Finally, the residual enthalpy may be calculated using

$$
H^{\mathrm{r}}(T, P, \underline{n})=A^{\mathrm{r}}-T\left(\frac{\partial A^{\mathrm{r}}}{\partial T}\right)_{V, \underline{n}}+P V-n_{\mathrm{T}} R T
$$

Several authors have presented the differences between the Debye-Hückel and MSA models for use in an equation of state. Myers et al. ${ }^{5}$ stated that MSA yields better results for the thermodynamic properties of electrolyte solutions at high concentrations. Along the same lines, Paricaud et al. ${ }^{19}$ stated that since the simpler Debye-Hückel theory treats ions as point charges it is only correct for infinitely dilute solutions and quickly breaks down at increasing electrolyte concentrations where the ion-ion and ion-solvent correlations become important. It has also been stated that the liquid densities are better described by MSA than Debye-Hückel. ${ }^{8,20}$ While the observations of these authors may be valid, it is important to realize that the liquid densities are obtained through an implicit function that includes volume and compositional derivatives from all contributions to the Helmholtz energy to solve for the bubble point pressure and vapor/liquid volumes. The results obtained from this procedure furthermore depend on the method used for parameter estimation and the experimental data that was used in the parameter estimation. Instead of analyzing the performance of the complete EoS, we restrict the analysis to the residual Helmholtz energy from MSA and Debye-Hückel at constant temperature, volume, and composition, to perform a fair comparison of MSA and DebyeHückel.

The following sections present the highlights of the derivations for illustrating the physical significance of the parameters $d_{i}$ and $\sigma_{i}$ of the two models. Following the discussion of the theoretical differences between the models, the paper presents the differences between the results for the Helmholtz energy and its first order derivatives with regards to temperature, volume, and composition.

\section{DISCUSSION AND RESULTS}

On the Derivation of the Debye-Hückel Theory. This section presents parts of the derivation of the Debye-Hückel theory to illustrate important concepts of electrolyte thermodynamics that are common to the Debye-Hückel and MSA theories for long-range electrostatic interactions. The derivation follows the derivation presented by other authors. $^{12,21-23}$

The Poisson equation relates the electrical potential $\psi_{i}$ of ion $i$ with the local charge density $c_{i}(r)$. In spherical coordinates, the Poisson equation may be written as

$$
\nabla^{2} \psi_{i}=-\frac{c_{i}(r)}{\varepsilon_{0} \varepsilon_{\mathrm{r}}}
$$

The local number density of the ions surrounding the central ion may be written as

$$
c_{i}(r)=\sum_{j}^{N} q_{j} \rho_{j} g_{i j}(r)
$$

where $g_{i j}(r)$ is the pair correlation function known from statistical mechanics ${ }^{21}$ and $\rho_{j}$ is the number density of component $j$. Equation 8 may only be solved when $g_{i j}(r)$ is a known function of the potential $\psi_{i}(r){ }^{24}$ To arrive at an expression for the pair correlation function, we define the potential of average force $w_{i j}(r)$ :

$$
g_{i j}(r)=\exp \left[-\frac{w_{i j}(r)}{k_{\mathrm{B}} T}\right]
$$

where $k_{\mathrm{B}}$ is the Boltzmann factor and $w_{i j}(r)$ is assumed to be proportional to the electrostatic potential:

$$
w_{i j}(r)=q_{j} \Psi_{i}(r)
$$

Inserting eq 10 into eq 9 gives an expression for the radial distribution function as shown in eq 11 .

$$
g_{i j}(r)=\exp \left[-\frac{q_{j} \psi_{i}(r)}{k_{\mathrm{B}} T}\right]
$$

Onsager $^{25}$ showed that the assumption that the distribution of ions was given by eq 11 becomes inconsistent for unsymmetrical electrolytes since the logical requirement that $\rho_{j} g_{j i}(r)=$ $\rho_{i} g_{i j}(r)$ is not fulfilled, except for the linearized Poisson equation. Onsager ${ }^{26}$ presented a procedure to remedy this discrepancy of the derivation by using higher order corrections of the Debye length, but this will not be investigated further here. The charge density function may be formed from eq 11 as shown in eq 12, and it follows that the Poisson equation is given by eq 13 :

$$
\begin{aligned}
& c_{i}(r)=\sum_{j}^{N} q_{j} \rho_{j} \exp \left[-\frac{q_{j} \Psi_{i}(r)}{k_{\mathrm{B}} T}\right] \\
& \nabla^{2} \Psi_{i}=-\frac{1}{\varepsilon_{0} \varepsilon_{\mathrm{r}}} \sum_{j}^{N} q_{j} \rho_{j} \exp \left[-\frac{q_{j} \Psi_{i}(r)}{k_{\mathrm{B}} T}\right]
\end{aligned}
$$


By linearizing eq 13 and using the electroneutrality condition, we arrive at

$$
\nabla^{2} \Psi_{i}=-\sum_{j}^{N} q_{j} \rho_{j}\left(1-\frac{q_{j} \psi_{i}(r)}{k_{\mathrm{B}} T}\right)
$$

Using the electroneutrality condition from eq 2, and introducing the Debye length $\kappa^{-1}$ as shown in eq 15 , we arrive at

$$
\begin{aligned}
& \kappa^{2}=\frac{1}{k_{\mathrm{B}} T} \frac{1}{\varepsilon_{\mathrm{r}} \varepsilon_{0}} \sum_{i}^{N} \rho_{i} q_{i}^{2} \\
& \nabla^{2} \Psi_{i}=\kappa^{2} \Psi_{i}(r)
\end{aligned}
$$

Thus, the electrical potential $\psi_{i}$ may be deduced by the solution to eq 16 imposing the boundary conditions $\lim _{r \rightarrow \infty} \psi_{i}(r)=0$ and from the Coulomb potential of the ion as presented in the derivation shown in, for example, McQuarrie $^{21}$ or Michelsen and Mollerup. ${ }^{22}$ With both models, the electrostatic potential of a molecule with fractional charge $\lambda q_{i}$ may be calculated using eq $17:^{22}$

$$
\psi_{i}\left(r=d_{i}\right)=\frac{\lambda q_{i}}{4 \pi \varepsilon_{r} \varepsilon_{0}} \frac{1}{d_{i j}} \frac{1}{1+\lambda \kappa d_{i j}}
$$

The total electrostatic potential at $r=d_{i j}$ may be related to the excess Helmholtz energy using the partial charging process, ${ }^{21,22}$ where the ion with charge $\lambda q_{i}$ is charged from $\lambda=0$ to 1 , as presented in eq 18 and 19:

$$
\begin{aligned}
& A^{\mathrm{DH}}=\sum_{i} n_{i} q_{i} \int_{0}^{1} \Psi_{i}(\lambda) \mathrm{d} \lambda \\
& A^{\mathrm{DH}}=-\frac{k_{\mathrm{B}} T V}{4 \pi N_{\mathrm{A}} \sum_{i} n_{i} z_{i}^{2}} \sum_{i} n_{i} z_{i}{ }^{2} \chi_{i}
\end{aligned}
$$

where the function $\chi_{i}$ is given by

$$
\chi_{i}=\frac{1}{d_{i}^{3}}\left[\ln \left(1+\kappa d_{i}\right)-\kappa d_{i}+\frac{1}{2}\left(\kappa d_{i}\right)^{2}\right]
$$

The complete derivatives of up to second order are provided in the book by Michelsen and Mollerup. ${ }^{22}$

Pitzer $^{27,28}$ discussed how the hard-core contribution could be included in the Debye-Hückel theory using a statistical mechanical treatment of the osmotic pressure $\Pi$ given by eq 21 .

$$
\Pi-\rho k_{\mathrm{B}} T=-\frac{4 \pi}{6} \sum_{i} \sum_{j} \rho_{i} \rho_{j} \int_{0}^{\infty}\left(\frac{\partial u_{i j}}{\partial r}\right) g_{i j}(r) r^{3} \mathrm{~d} r
$$

Using a potential $u_{i j}$ for the hard-core ions given by eq 22 , Pitzer $^{27}$ derived the expression shown in eq 23 by including the third order Taylor expansion of the radial distribution function $g_{i j}$ from eq 11 .

$$
u_{i j}= \begin{cases}\infty & r<d_{i j} \\ \frac{z_{i} z_{j} e^{2}}{4 \pi \varepsilon_{\mathrm{r}} \varepsilon_{0} r} & r \geq d_{i j}\end{cases}
$$

$$
\begin{aligned}
\frac{\Pi}{\rho k_{\mathrm{B}} T}-1= & -\frac{\kappa^{3}}{24 \pi \rho\left(1+\kappa d_{i}\right)} \\
& +\rho\left[\frac{2 \pi d^{3}}{3}+\frac{1}{48 \pi} \frac{\kappa^{4} d_{i}}{\rho^{2}\left(1+\kappa d_{i}\right)^{2}}\right]
\end{aligned}
$$

As pointed out by Onsager, ${ }^{22}$ methods that use higher order terms of the radial distribution function will become inconsistent for unsymmetrical electrolytes as the logical requirement $\rho_{j} g_{j i}(r)=\rho_{i} g_{i j}(r)$ is not fulfilled. Pitzer notes that the method is consistent for symmetrical electrolytes, and that the inconsistency for unsymmetrical cases is small. Since the model by Pitzer includes the contribution from hard-core repulsions, it is not suited as a perturbation for the electrostatic forces in an EoS, as the EoS already contains terms that account for the short-range interactions.

On the Derivation of the MSA Theory. The fundamental difference between the derivations of the MSA and the DebyeHückel theories is that MSA treats the ions as hard-core spherical molecules with diameter $\sigma_{i}$. This gives rise to an excluded volume that is inaccessible to other ions leading to different expressions for the Helmholtz energy and screening length compared to the Debye-Hückel theory.

The lengthy derivation of MSA has been presented by Blum $^{13,14}$ and will not be repeated here. The final expression for the excess internal energy is given by eq 24 :

$$
\Delta E^{\mathrm{MSA}}=-\frac{V e^{2}}{4 \pi \varepsilon_{\mathrm{r}} \varepsilon_{0}} \sum_{i}^{N} \rho_{i} z_{i}\left[\frac{\Gamma z_{i}+\eta \sigma_{i}}{1+\sigma_{i} \Gamma}\right]
$$

where $z_{i}$ is the charge of the ion, $\Gamma$ is given by the MSA closure equation from eq 25 , and the parameter $\eta$ is calculated from

$$
\begin{aligned}
& (2 \Gamma)^{2}=\frac{1}{k_{\mathrm{B}} T} \frac{e^{2}}{\varepsilon_{0} \varepsilon_{\mathrm{r}}} \sum_{k} \rho_{k}\left(z_{k}+N_{k} \sigma_{k}\right)^{2} \\
& \eta=\frac{1}{\Omega} \frac{\pi}{2 \Delta} \sum_{k} \frac{\rho_{k} \sigma_{k} z_{k}}{1+\Gamma \sigma_{k}}=\frac{1}{\Omega} \frac{\pi}{2 \Delta} \frac{N_{\mathrm{A}}}{V} \sum_{k} \frac{n_{k} \sigma_{k} z_{k}}{1+\Gamma \sigma_{k}}
\end{aligned}
$$

The distance $(2 \Gamma)^{-1}$ is the MSA equivalent of the screening length in the Debye-Hückel theory $\kappa^{-1}$, and the auxiliary function $\Omega$ is calculated using

$$
\Omega=1+\frac{\pi}{2 \Delta} \sum_{k} \frac{\rho_{k} \sigma_{k}^{3}}{1+\Gamma \sigma_{k}}=1+\frac{\pi}{2 \Delta} \frac{N_{\mathrm{A}}}{V} \sum_{k} \frac{n_{k} \sigma_{k}^{3}}{1+\Gamma \sigma_{k}}
$$

$\Delta$ is a measure of the included volume calculated using eq 28 and must be strictly positive and larger than $\sim 0.26$ (the facecentered cubic packing factor). Note that this term also includes the contribution from uncharged species in the mixture:

$$
\Delta=1-\frac{\pi}{6} \sum_{k} \rho_{k} \sigma_{k}^{3}=1-\frac{\pi}{6} \frac{N_{\mathrm{A}}}{V} \sum_{k} n_{k} \sigma_{k}^{3}
$$

The Helmholtz free energy may be calculated using the thermodynamic relation as follows:

$$
\frac{\partial}{\partial\left(k_{\mathrm{B}} T\right)^{-1}}\left(\frac{A^{\mathrm{MSA}}}{k_{\mathrm{B}} T}\right)=\Delta E^{\mathrm{MSA}}
$$


The integration shown in eq 29 cannot be performed directly, but we may use the method by Høye and Stell, ${ }^{29}$ where the Helmholtz energy may be expressed from

$$
\frac{A^{\mathrm{MSA}}}{k_{\mathrm{B}} T}=\frac{\Delta E^{\mathrm{MSA}}}{k_{\mathrm{B}} T}-\int_{0}^{\Gamma} \mathrm{d} \Gamma^{\prime}\left(\frac{\partial}{\partial \Gamma^{\prime}} \frac{\Delta E^{\mathrm{MSA}}}{k T}\right)
$$

Note that taking the derivative of eq 30 with respect to $\Gamma$ yields the identity in eq 31 :

$$
\frac{\partial}{\partial \Gamma}\left(\frac{A^{\mathrm{MSA}}}{k_{\mathrm{B}} T}\right)_{T, V, n}=0
$$

The solution to the integral in eq 30 gives the surprisingly simple result

$$
\frac{A^{\mathrm{MSA}}}{k_{\mathrm{B}} T}=\frac{V \Gamma^{3}}{3 \pi}-\frac{1}{k_{\mathrm{B}} T} \frac{V e^{2}}{4 \pi \varepsilon_{\mathrm{r}} \varepsilon_{0}} \times \sum_{i}^{N} \rho_{i} z_{i}\left[\frac{\Gamma z_{i}+\eta \sigma_{i}}{1+\sigma_{i} \Gamma}\right]
$$

Furthermore, as we have eq 31 , we may deduce another form of eq 25:

$$
(2 \Gamma)^{2}=\frac{1}{k_{\mathrm{B}} T} \frac{e^{2}}{\varepsilon_{\mathrm{r}} \varepsilon_{0}} \sum_{i}^{N} \rho_{i} z_{i} \frac{\partial}{\partial \Gamma}\left[\frac{\Gamma z_{i}+\eta \sigma_{i}}{1+\sigma_{i} \Gamma}\right]
$$

The MSA theory has one internal variable $\Gamma$ which is not known from explicit relations and must be obtained from numerical methods. The Supporting Information contains a fast and robust iterative scheme for solving eq 33 based on second order minimization.

The Electrostatic Moment Conditions. The solutions to the four first moments are interesting in terms of analyzing the importance of the assumptions done in the Debye-Hückel theory, that is, that the radial distribution function is only a function of the long-range forces from the electrostatic interactions. From the Coulombic forces between ions (eq 1) it is possible to derive a set of statistical mechanical moment conditions for electrolytes using the Ornstein-Zernike equation. A derivation of the moment conditions has been presented by, for example, Attard, ${ }^{23}$ and his results are summarized in this paper to illustrate that the Debye-Hückel theory cannot satisfy the fourth moment condition due to neglecting the short-range forces. The zeroth moment condition is shown in eq 34 :

$$
4 \pi \sum_{k} \rho_{k} z_{k} \int_{0}^{\infty} r^{2} h_{i k}(r) \mathrm{d} r=-z_{i}
$$

The left-hand side of eq 34 represents integration over all ions in the vicinity of the central ion. The physical significance of eq 34 is that the total charge in the ion cloud surrounding the central ion $i$ will be of same magnitude but opposite charge as the central ion $i{ }^{14}$ The second moment is thus the statistical mechanical equivalent of the electroneutrality condition from eq 2 .

The Stillinger-Lovett second moment condition is given by eq 35:

$$
\frac{4 \pi e^{2}}{\varepsilon_{0} \varepsilon_{r} k_{\mathrm{B}} T} \sum_{i} \sum_{j} \rho_{i} \rho_{j} z_{i} z_{j} \times \int_{0}^{\infty} r^{4} h_{i k}(r) \mathrm{d} r=-6
$$

The physical significance of the Stillinger-Lovett second moment is that for finite size ions, a charge oscillation occurs in the ionic cloud. ${ }^{23}$ Note that neither the zeroth nor the second moment depend on the short-range interactions.

The fourth moment is shown in eq $36:{ }^{23}$

$$
\frac{1}{120} \mathbf{Q} \mathbf{H}^{(4)} \mathbf{Q}=\left(\mathbf{H}^{(0)}-\mathbf{C}^{(0)}-\mathbf{H}^{(0)} \mathbf{C}^{(0)}\right)\left(\mathbf{I}-\mathbf{C}^{(0)}\right)
$$

where the matrix $\mathbf{Q}$ is given by eq $37, \mathbf{H}^{(0)}$ is from the first term of the linearization of the indirect correlation function $h_{i j}(r)$ in eq 38 , and $\mathbf{C}^{(0)}$ is from the first term of the linearization of the short-range part of the direct correlation function as given by eq 39

$$
\begin{aligned}
& \mathbf{Q}_{i j}=\frac{\sqrt{\rho_{i} \rho_{j}}}{k_{\mathrm{B}} T} \frac{q_{i} q_{j}}{\varepsilon_{0} \varepsilon_{\mathrm{r}}} \\
& \mathbf{H}_{i j}{ }^{0}=\left(\rho_{i} \rho_{j}\right)^{1 / 2}{h_{i j}}^{0}(\mathbf{r}) \\
& \mathbf{C}_{i j}{ }^{0}=\left(\rho_{i} \rho_{j}\right)^{1 / 2} c_{i j}{ }^{0}(\mathbf{r})
\end{aligned}
$$

It is evident that the fourth moment depends on the shortrange interactions, represented by $\mathbf{C}^{(0)}$. The fourth moment condition has been related to the partial ionic structure factors and the isothermal compressibility of the electrolyte. ${ }^{30,31}$ The Debye-Hückel theory assumes that the short-range interactions are negligible, that is, that $\mathbf{C}^{(0)}=0$, but the MSA accounts for the short-range interactions by treating the ions as hard spheres.

On the Relationship between EoS and Activity Coefficient Models. An EoS will include one of the terms for the Helmholtz energy from Debye-Hückel (eq 19) or MSA (eq 32) to account for the long-range electrostatic interactions, as a perturbation to the reference EoS. Insofar as the efforts to form a working EoS for mixtures with electrolytes have not resulted in significant improvements over the activity coefficient (or excess Gibbs energy) models for electrolyte systems, ${ }^{1}$ this section serves to present the relationship between the expressions for the long-range electrostatic forces developed for the Debye-Hückel and MSA theories, and the activity coefficient models that are widely used by the industry to predict thermodynamic properties of mixtures containing electrolytes.

The chemical potential may be determined from the compositional derivative of either the Helmholtz or the Gibbs free energy:

$$
\mu_{i}=\left(\frac{\partial A}{\partial n_{i}}\right)_{T, V, n_{j \neq i}}=\left(\frac{\partial G}{\partial n_{i}}\right)_{T, P, n_{j \neq i}}
$$

The chemical potential may be split into two terms from the relation $\mu_{i}=\mu_{i}^{\mathrm{i}}+\mu_{i}^{\mathrm{E}}$, one term corresponding to the ideal mixture contribution $\mu_{i}^{\mathrm{i}}$, and one term corresponding to the excess chemical potential $\mu_{i}^{\mathrm{E}}$. The activity coefficient may then be obtained from the excess chemical potential using the wellknown relation $\ln \gamma_{i}=\mu_{i}^{\mathrm{E}} /(R T)$. The chemical potential may be calculated from either the rational (mole-fraction) or the molality scale, and may furthermore be given as the unsymmetrical activity coefficient, tending to 1 as the concentration goes to zero. Commonly, activity coefficient models for electrolyte mixtures use unsymmetrical activity coefficients using the molality 
scale for solutes, and the symmetrical rational activity coefficient for solvents.

Debye and Hückel ${ }^{12}$ originally derived a model for the activity coefficient from the expression of the excess Helmholtz energy from eq 19 by replacing the molar volume and static permittivity by empirical correlations, and using the resulting equation as an expression for the excess Gibbs energy. This procedure has been used for activity coefficient models. ${ }^{2,3,36,37}$ Electrolyte equations of state determine the volume from the pressure equation given by eq 4. Primitive electrolyte equations of state ${ }^{4-10,18-20,24}$ determine the static permittivity from (semi)-empirical solvent-specific correlations while nonprimitive equations of state ${ }^{11}$ determine the static permittivity from dipolar interactions. Common to both the electrolyte EoS and activity coefficient models is that they include terms that account for the short-range interactions; activity coefficient models determine the short-range forces from local-composition models as UNIQUAC ${ }^{2}$ or NRTL, ${ }^{3}$ or from Pitzer's modifications of the Debye-Hückel theory to include a hardcore repulsive term shown in eq 23.

Numerical Comparison of the Debye-Hückel and MSA Theories. The previous section introduced the DebyeHuckel and MSA models and showed the major differences between the assumptions of these two models. It is of interest to investigate the numerical differences in the excess Helmholtz energy to determine the differences between MSA and DebyeHückel. It was decided to use the same numerical value of the ion diameter in MSA as in Debye-Hückel to provide a basis for comparing the two models, thus in this section, $\sigma_{i}$ will be used as the symbol for the ion diameter and the distance of closest approach $d_{i}$. Typical Pauling radii are shown in Table 1.

Table 1. Pauling Radii for Selected Ions ${ }^{32}$ (Marcus, 1988)

\begin{tabular}{cccccccc} 
ion & $\mathrm{Li}^{+}$ & $\mathrm{Na}^{+}$ & $\mathrm{Mg}^{2+}$ & $\mathrm{Ca}^{2+}$ & $\mathrm{Cl}^{-}$ & $\mathrm{SO}_{4}^{2-}$ & $\mathrm{NO}_{3}^{-}$ \\
radii $[\AA]$ & 0.74 & 1.02 & 0.72 & 1.00 & 1.81 & 2.30 & 1.79 \\
\hline
\end{tabular}

To obtain the complete picture of the differences between the two models, it was decided to vary the following model parameters: (i) ion diameter, (ii) ion charge, (iii) temperature, (iv) volume, (v) composition. Additionally, as has been shown by several authors, $5,7,18,22,33,34$ the relative static permittivity or dielectric constant $\varepsilon_{\mathrm{r}}$ is a function of temperature, volume, and composition, and that this dependence is of high importance to the performance of the electrolyte equation of state. To determine the influence of the static permittivity on the performance of the two models, the model presented by Michelsen and Mollerup $^{22}$ was implemented. According to this model, the relative static permittivity is calculated using the empirical expression

$$
\varepsilon_{\mathrm{r}}(T, V, \underline{n})=\varepsilon_{r}^{\mathrm{w}}(T) \times E(\mathbf{n}, V)
$$

where $E(\mathbf{n}, V)$ is an ion correction factor that effectively serves to reduce the relative static permittivity. $\varepsilon_{r}^{\mathrm{w}}$ is the relative static permittivity of water calculated as a function of temperature:

$$
\varepsilon_{\mathrm{r}}^{\mathrm{w}}(T)=\varepsilon_{\mathrm{r}}^{\mathrm{w}, 0}\left(T_{0}+\frac{\beta_{1}}{2} \frac{N_{A} \mu_{\mathrm{w}}^{2}}{k_{\mathrm{B}} \varepsilon_{0}}\left(\frac{\rho_{\mathrm{w}}(T)}{T}-\frac{\rho_{\mathrm{w}}\left(T_{0}\right)}{T_{0}}\right)\right)
$$

where $T_{0}=273.15 \mathrm{~K}$ is the reference temperature, $\varepsilon_{r}^{\mathrm{w}, 0}=87.82$ is the relative static permittivity at $T_{0}, \mu_{\mathrm{w}}=8.33 \times 10^{-30} \mathrm{C} \cdot \mathrm{m}$ is the dipole moment of water in vacuum, $N_{\mathrm{A}}$ is the Avogadro number, and $\beta_{1}=3.1306$ is a fitting constant. The density of water $\rho_{\mathrm{w}}(T)$ was calculated using

$$
\rho_{\mathrm{w}}(T)=\rho_{0}+\omega_{1}\left(T-T_{0}\right)+\omega_{2}\left(T-T_{0}\right)^{\omega_{3}}
$$

where $\rho_{0}=0.99984 \mathrm{~kg} / \mathrm{L}, \omega_{1}=1.51782 \times 10^{-4} \mathrm{~kg} /(\mathrm{L} \cdot \mathrm{K}), \omega_{2}=$ $-4.50573 \times 10^{-5} \mathrm{~kg} /\left(\mathrm{L} \cdot \mathrm{K}^{\omega_{3}}\right)$ and $\omega_{3}=1.55$.

The $E$-factor is given by

$$
E(\mathbf{n}, V)=1+\sum_{k}^{\text {ions }}\left(\beta_{3} c_{k}-\frac{\alpha_{k} c_{k}}{1+\beta_{2} c_{k}}\right)
$$

where $\beta_{2}=0.160 \mathrm{~L} / \mathrm{mol}, \beta_{3}=0.010 \mathrm{~L} / \mathrm{mol}, c_{k}$ is the concentration of species $k$, and $\alpha_{k}$ is an ion specific constant. Selected values are shown in Table 2, and an example calculation for the static permittivity of $\mathrm{NaCl}$ and $\mathrm{MgCl}_{2}$ are presented in Figure 3. The relative static permittivity and density of pure water are shown in Figure 2.

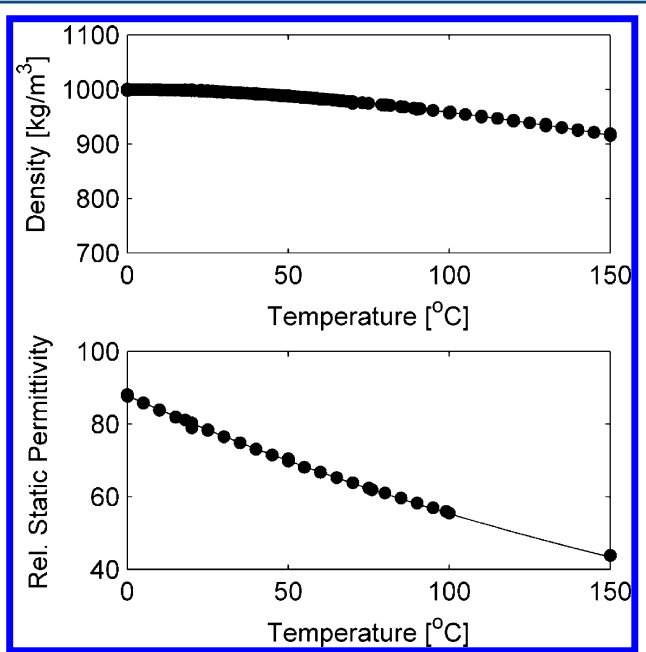

Figure 2. Temperature dependence of relative static permittivity and liquid density for water using eqs 42 and 43. Experimental data indicated by the filled circle $(\bullet)$ are from the Landolt Börnstein database. $^{35}$

If not stated otherwise, we will use a constant volume and the static permittivity of pure water as the basis of the comparison, and therefore not generally include the effect of the ions on the static permittivity. This is done to more easily distinguish the effect of parameters on the models. The first section presents the important results of the numerical investigation on the effect of the screening length, while the following section

Table 2. Ion Specific Parameters for Calculation of the Dielectric Constant ${ }^{22}$

$\begin{array}{ccccccc}\text { ion } & \mathrm{Li}^{+} & \mathrm{Na}^{+} & \mathrm{Mg}^{2+} & \mathrm{Ca}^{2+} & \mathrm{Cl}^{-} & \mathrm{SO}_{4}^{2-} \\ \text { correction factor }[\mathrm{L} / \mathrm{mol}] & 0.12 & 0.1062 & 0.1155 & 0.1097 & 0.1173 & 0.0022\end{array}$




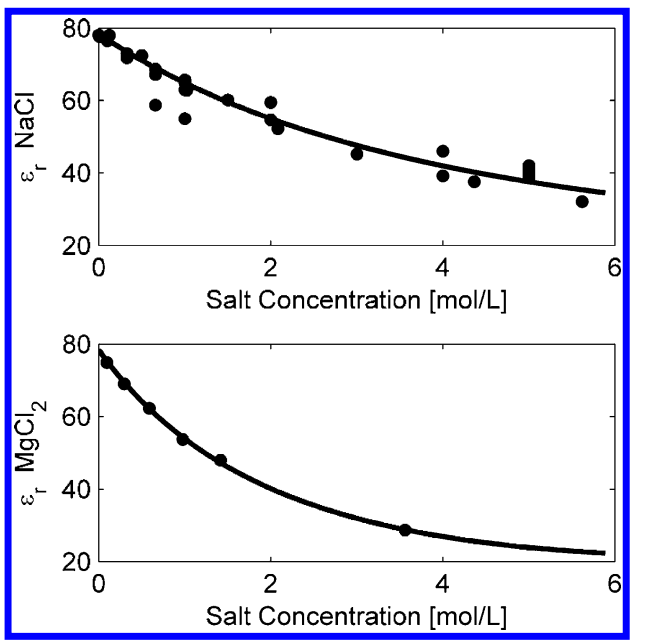

Figure 3. Effect of salt concentration on relative static permittivity of $\mathrm{NaCl}$ and $\mathrm{MgCl}_{2}$ using eq 44. Experimental data indicated by the filled circle $(\bullet)$ is taken from the collections of Akhadov ${ }^{36}$ and Barthel et al. ${ }^{37}$

presents the influence on the Helmholtz energy and its first order derivatives.

Comparison of Screening Length. A comparison of eq 15 to eq 25 shows that the screening length in the DebyeHückel theory is independent of diameter, while this is not true for MSA. Figure 4 compares the screening length of MSA and Debye-Hückel at $25^{\circ} \mathrm{C}$.

As shown in Figure 4 the Debye-Hückel theory predicts an unphysical behavior where increasing the ion size does not increase the screening length. The difference between the screening length in MSA and Debye-Hückel depends on the molality and the ion diameter.

When the volume and static permittivity are kept constant, the screening length of the Debye-Hückel and MSA display a linear dependence on temperature, that is, the higher the temperature is, the higher is the screening length. The temperature dependence of the screening length at $3 \mathrm{~m}$ and constant volume is shown in Figure 5:

Figure 5 shows that the screening lengths of the two models display a similar and weak temperature dependence.

The volume does change in the presence of electrolytes, while this effect was not included in either Figure 4 or Figure 5, as the comparisons were carried out at constant volume, and

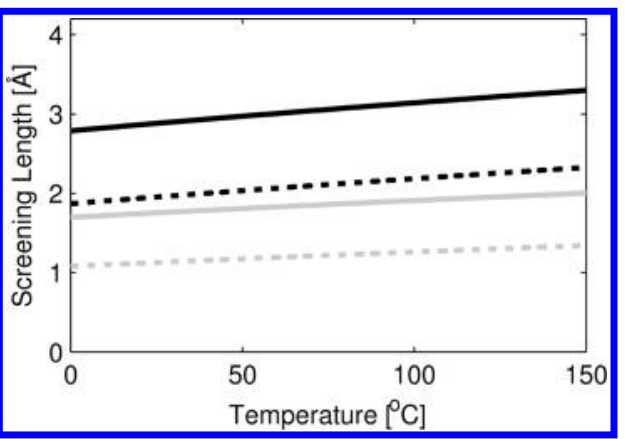

Figure 5. Comparison of the temperature-dependence of the screening lengths calculated with MSA (solid) and Debye-Hückel (dashed) for $\mathrm{NaCl}$ (black) and $\mathrm{MgCl}_{2}$ (gray) at $25^{\circ} \mathrm{C}, 3 \mathrm{~m}$, and $1.2 \mathrm{~L}$ volume with relative static permittivity $\varepsilon_{\mathrm{r}}=78$.

not constant pressure. Figure 6 illustrates the influence of volume on the screening length:

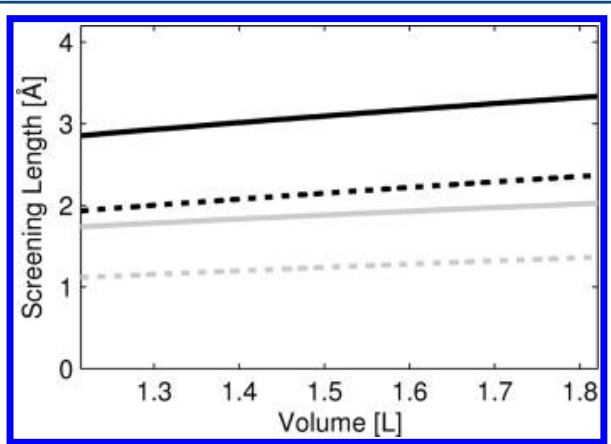

Figure 6. Comparison of volume-dependence of the screening length calculated by MSA (solid) and Debye-Hückel (dashed) for $\mathrm{NaCl}$ (black) and $\mathrm{MgCl}_{2}$ (gray) at $25{ }^{\circ} \mathrm{C}, 3 \mathrm{~m}$, and relative static permittivity $\varepsilon_{\mathrm{r}}=78$.

Figure 6 illustrates that the screening length is proportional to the volume, but also that the volume dependence of the screening lengths in the two models is rather weak. Figure 7 illustrates the influence of the relative static permittivity on the screening length.

Figure 7 shows that both models show similar dependence of the screening length on the relative static permittivity. A decrease
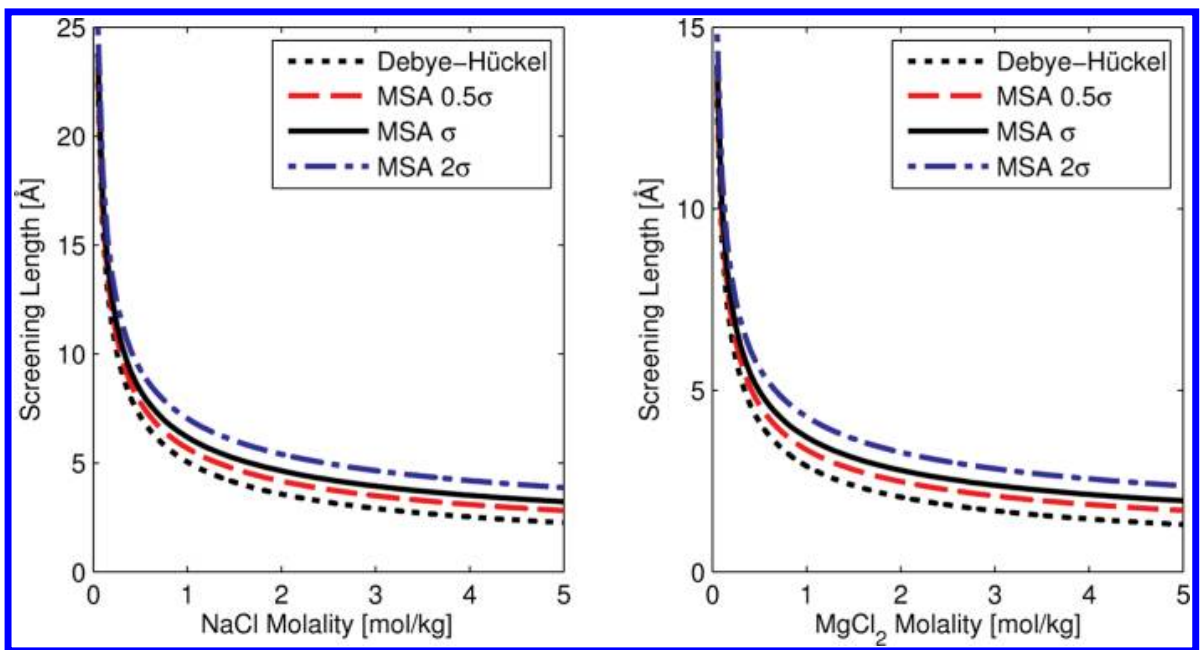

Figure 4. Comparison of screening length at $25^{\circ} \mathrm{C}$, a $2.8 \mathrm{~L}$ volume, and constant relative static permittivity $\varepsilon_{\mathrm{r}}=78$. 


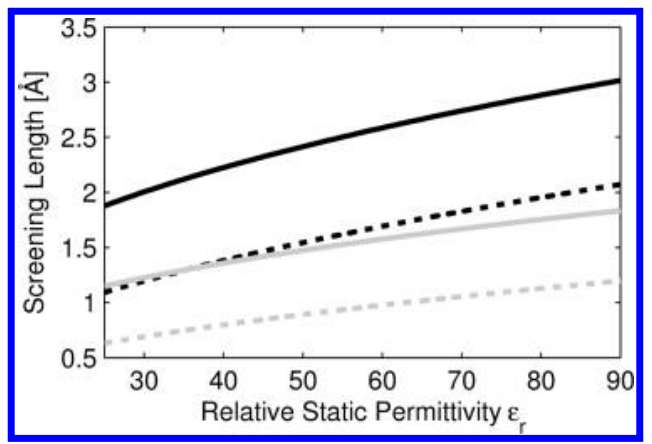

Figure 7. Comparison of the dependence of the screening lengths on the static permittivity calculated by MSA(solid) and Debye-Hückel (dashed) for $\mathrm{NaCl}$ (black) and $\mathrm{MgCl}_{2}$ (gray) at $3 \mathrm{~m}$, constant volume at $1 \mathrm{~L}$, and constant temperature at $25^{\circ} \mathrm{C}$.

in the static permittivity caused by the compositional changes or due to an increase in the temperature will yield a decrease in the screening length. Owing to its typical range of values in aqueous electrolytes as presented in Figure 3, the static permittivity has a larger influence on the screening lengths than temperature and volume alone.

The two models display the same trends for the screening length when changing the temperature, volume, ion charges, composition, and static permittivity. However, only MSA predicts an increase in the screening length with increasing ion diameters. We can therefore consider MSA to be a better choice in cases where the numerical value of the screening length is important (e.g., for interfacial phenomena).

Comparison of Helmholtz Energy. The Helmholtz energy and its derivatives are of great importance for equations of state that incorporate either MSA or Debye-Hückel to account for the ion-ion interactions. This section compares the trends predicted by the two models using the same procedure as presented in the previous section.

Effect of Ion Diameter. Although the ion diameter does not influence the screening length in the Debye-Hückel theory, it is important for the calculation of the Helmholtz energy. Figure 8 compares the Helmholtz energy using the Debye-Hückel and MSA models for different ion diameters.
Figure 8 and Figure 4 show that an increase in the ion size leads to an increase in the separation distance between the ions in both models, thereby reducing the interaction energy and thus the Helmholtz energy. In all cases MSA yields lower energies at the same concentration. The larger excluded volume at higher concentrations contributes to lowering the Helmholtz energy in the case of MSA, whereas the Debye-Hückel theory does not account for this effect. Since the Helmholtz energy from the Debye-Hückel theory is always slightly larger than the Helmholtz energy calculated using MSA, we may speculate that choosing an empirical modification of the ion diameter in the Debye-Hückel and MSA theories as shown in eq 45, would lead to identical results from the two models:

$$
d_{i}=\omega \sigma_{i}
$$

Interestingly, when choosing $\omega=5 / 6$, the values of the Helmholtz energies and their derivatives from both models become similar (within 5\%) for different ion sizes, ion charges, and temperatures up to $5 \mathrm{~m}$. The largest deviations are observed in the volume derivative for large ions. It was furthermore found that the size of the solvent does not change the optimal $\omega$ factor significantly, but it does have an effect on the value of the volume derivative. Since the MSA theory accounts for the excluded volume explicitely and Debye-Hückel does not, the physical significance of the $\omega$ factor is to account for the effect of the excluded volume by reducing the separation distance of the ions. From this analysis, it may be concluded that if the ion size is fitted during pure component parameter estimation, the two models can perform similarly.

When the static permittivity is calculated from the composition using the empirical model given by eq 41 , the reduced Helmholtz energy changes by $50-100 \%$ as evidenced by Figure 9 .

Figure 9 shows that the static permittivity has a profound effect on the reduced Helmholtz energy, and in the following sections the influence of using the empirical model on the derivatives of the residual Helmholtz energy is investigated.

Comparison of Volume Derivative. Figure 10 presents the influence of volume, ion charge, and ion size on the volume derivative:

Figure 10 shows that the volume derivative of Helmholtz energy increases with increasing ion concentration and temperature. Figure 10 does not include the effect of volume on the dielectric constant, as this is not included in the

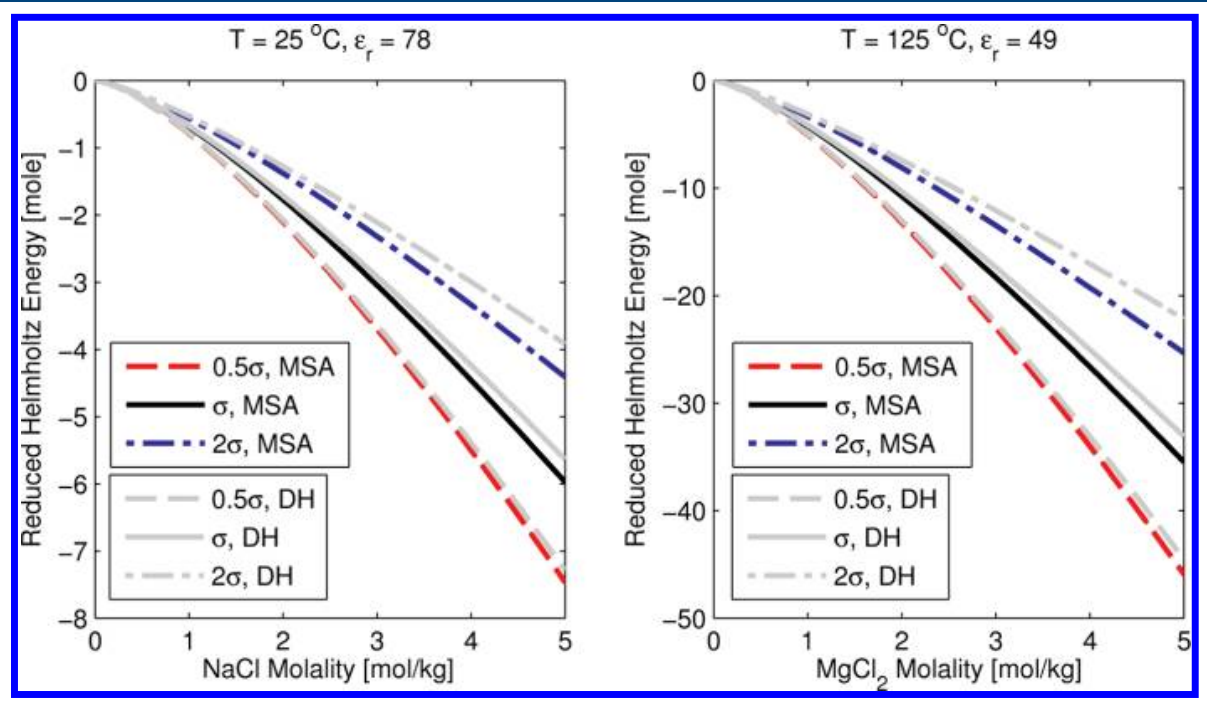

Figure 8. Comparison of the Helmholtz energy at different diameters with relative static permittivity equal to that of water (shown above the panels). Volume is fixed at $2.8 \mathrm{~L}$. 

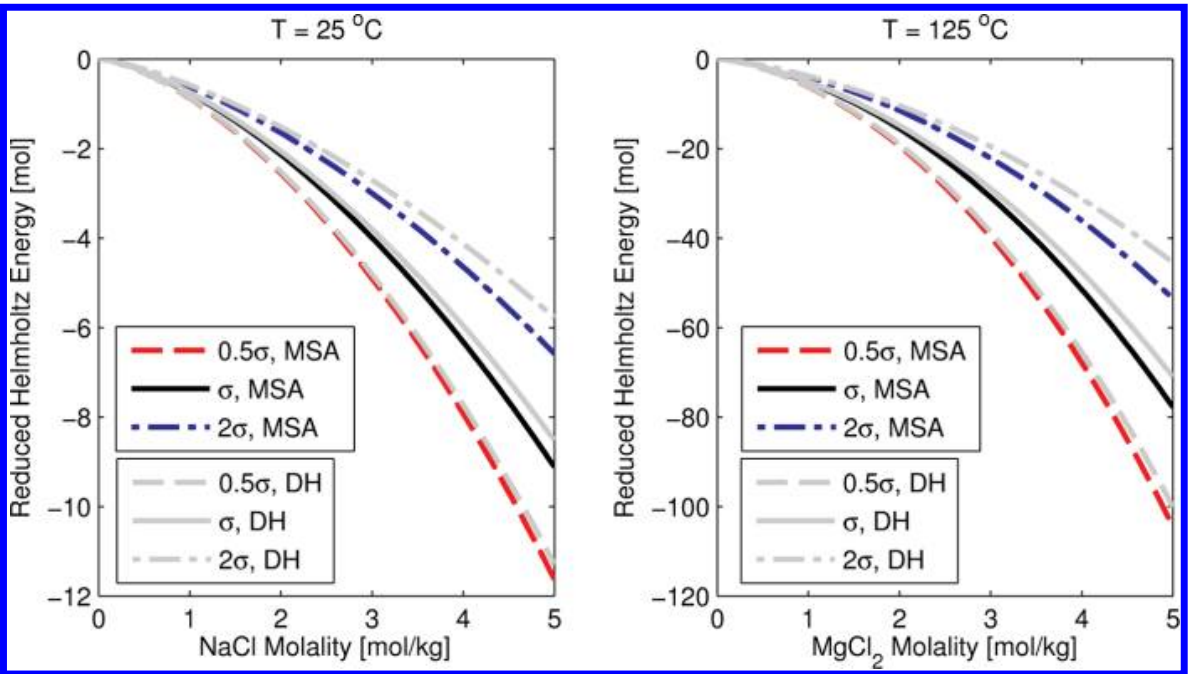

Figure 9. Comparison of the Helmholtz energy at different diameters with composition dependent static permittivity. Volume is fixed at $2.8 \mathrm{~L}$.
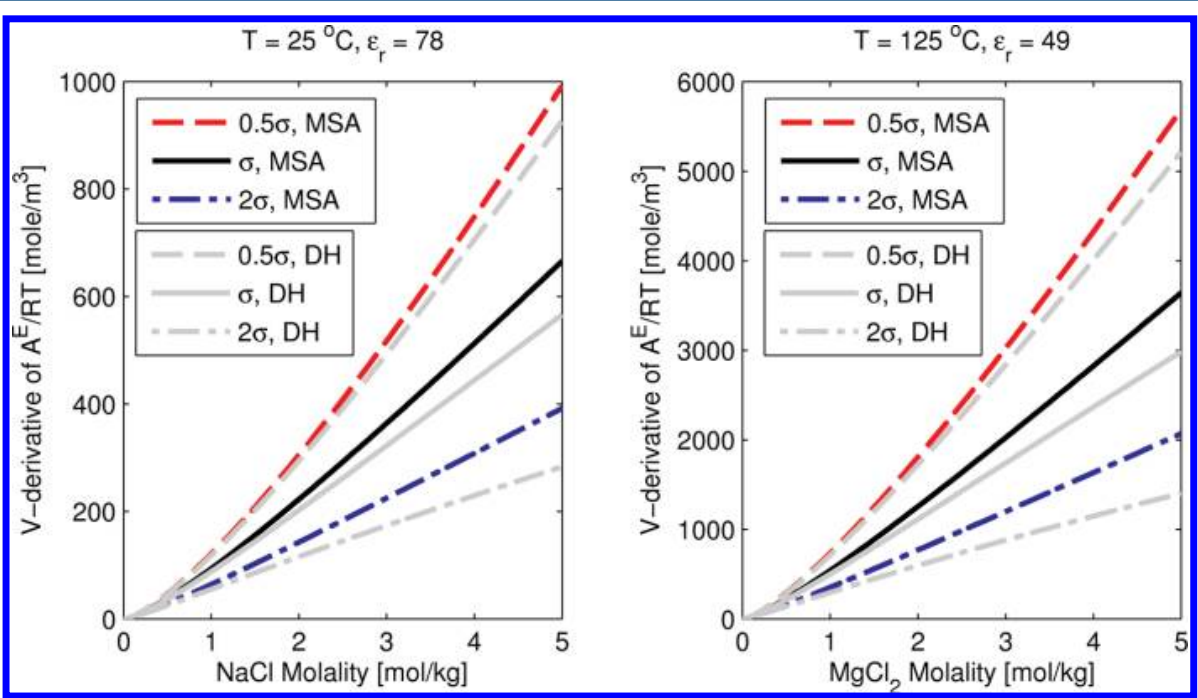

Figure 10. Comparison of the volume derivative of Helmholtz energy at different diameters at constant temperature and relative static permittivity (shown above the figure). Volume is fixed at $2.8 \mathrm{~L}$.
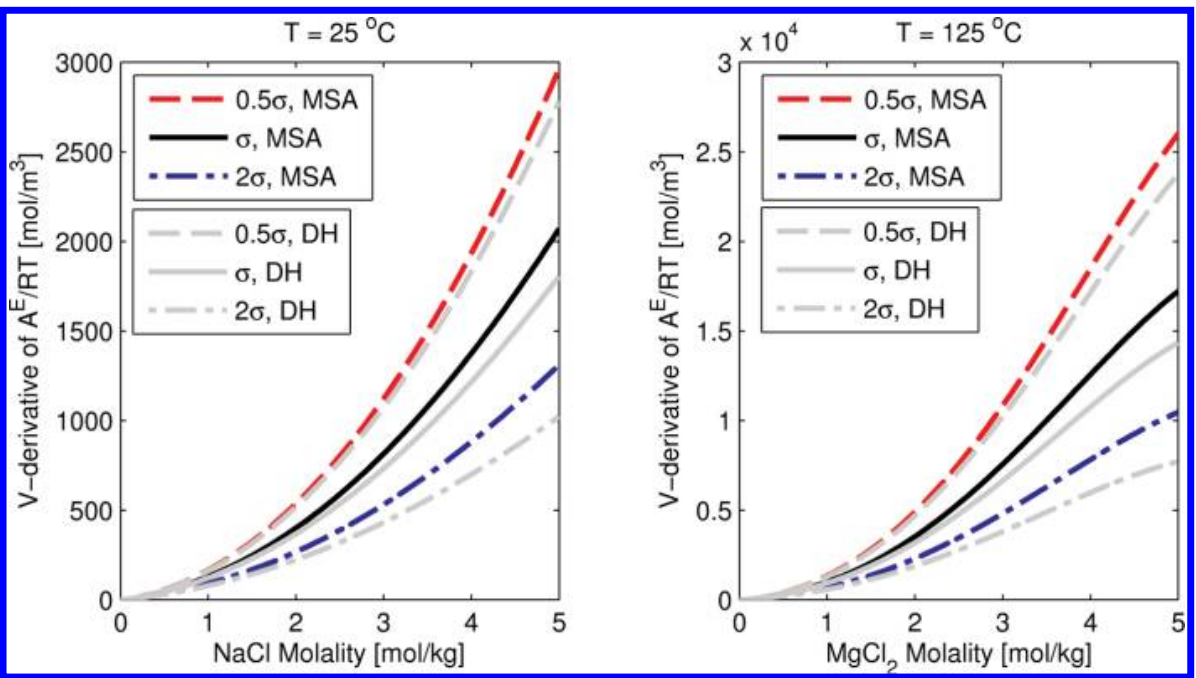

Figure 11. Comparison of the volume derivative of Helmholtz energy when eq 41 is used to model the relative static permittivity. Volume is fixed at $2.8 \mathrm{~L}$.

empirical model for the static permittivity of pure water given by eq 42 . However, if we would use the model for the static permittivity given by eq 41 the volume derivatives are affected as shown in Figure 11. 


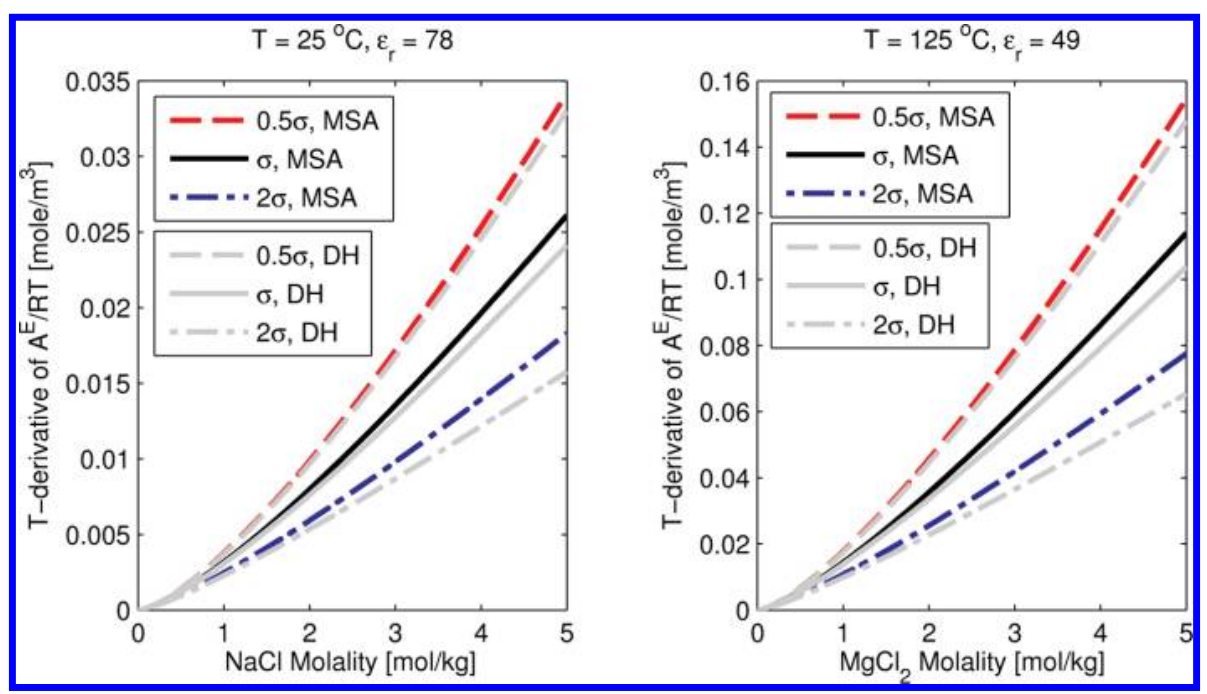

Figure 12. Comparison of temperature derivative of Helmholtz energy at different ion diameters. The temperature derivative of the static permittivity of pure water was set to zero to observe the behavior of the model without including the temperature dependence of the static permittivity. MSA is black colored, Debye-Hückel is gray. Volume is fixed at $2.8 \mathrm{~L}$.

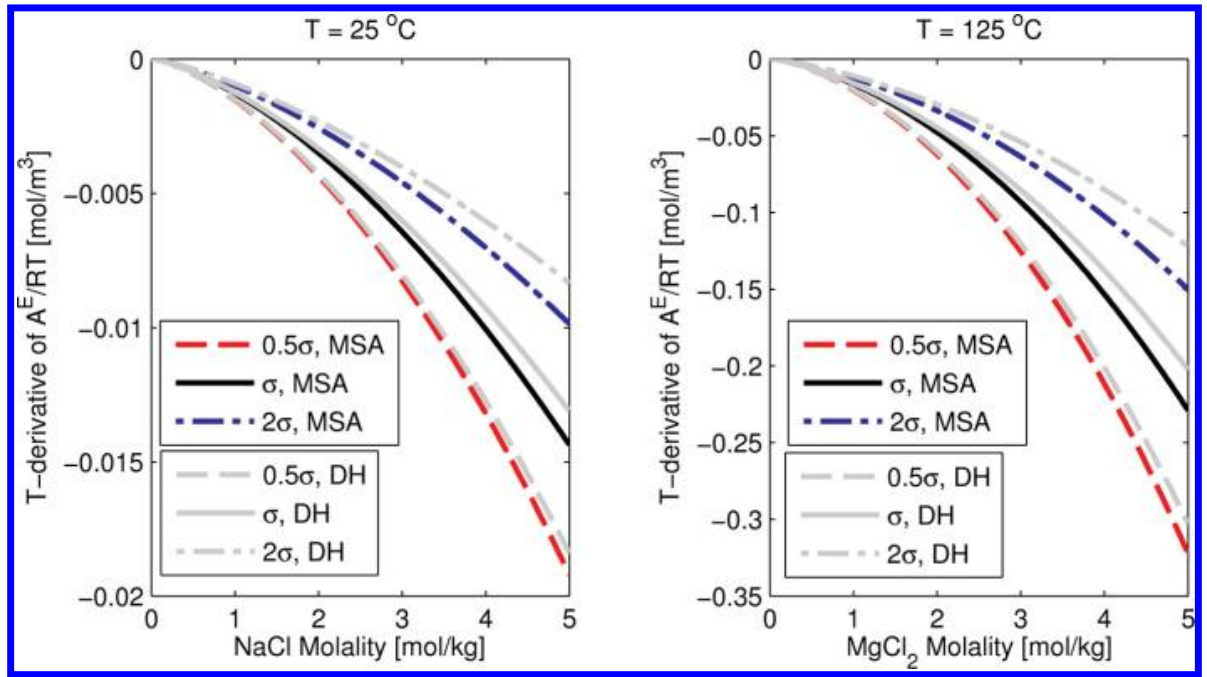

Figure 13. Comparison of temperature derivative of Helmholtz energy at different ion diameters using the empirical correlation for the relative static permittivity shown in eq 41 . Volume is fixed at $2.8 \mathrm{~L}$.

As the volume derivative is inversely dependent on the static permittivity, the reduction of $\varepsilon_{\mathrm{r}}$ due to the presence of salts serves to increase the volume derivative. Additionally a volume dependency is introduced through the $E(\mathbf{n}, V)$ correction function given by eq 44 . Figure 11 illustrates that accounting for the effect of salts has a substantial effect on the volume derivative. However, the result shown in Figure 11 is not necessarily the correct physical behavior, but rather a result of fitting the empirical model parameters to concentration data (see eq 44). If the correction factor was depending on molality or mole fractions rather than concentrations, the volume dependency would become zero, which would influenced the behavior shown in Figure 11. Extreme care should be exercised when using empirical correlations for the static permittivity, as they may end up including a nonphysical behavior of the Helmholtz energy expression of the electrostatic models. It is considered of high importance to have a model for the permittivity that not only fits the data but actually resembles the correct physical dependence on temperature, volume, and composition.
Comparison of Temperature Derivative. The influence of temperature, ion charge, and ion size on the temperature derivative is presented in Figure 12.

The same trend is observed for both MSA and DebyeHückel in Figure 12 where the absolute value of the temperature derivative increases with increasing ion size and temperature for both models. Figure 13 shows that when the temperature derivative of the static permittivity is included together with the empirical correlation for the static permittivity, the temperature derivative changes sign but is of the same order of magnitude.

Comparison of Compositional Derivatives. As shown in eq 5 , the fugacity coefficient depends on the compositional derivatives of the Helmholtz energy. The fugacity coefficients are used in the calculation of the thermodynamic equilibrium properties at constant temperature and pressure. In an EoS, the fugacity coefficients at constant pressure are determined by first solving for the volume root from the pressure specified in eq 4 and then inserting this volume into eq 5 . In Figure 14, we analyze the contribution to the Helmholtz energy from MSA 


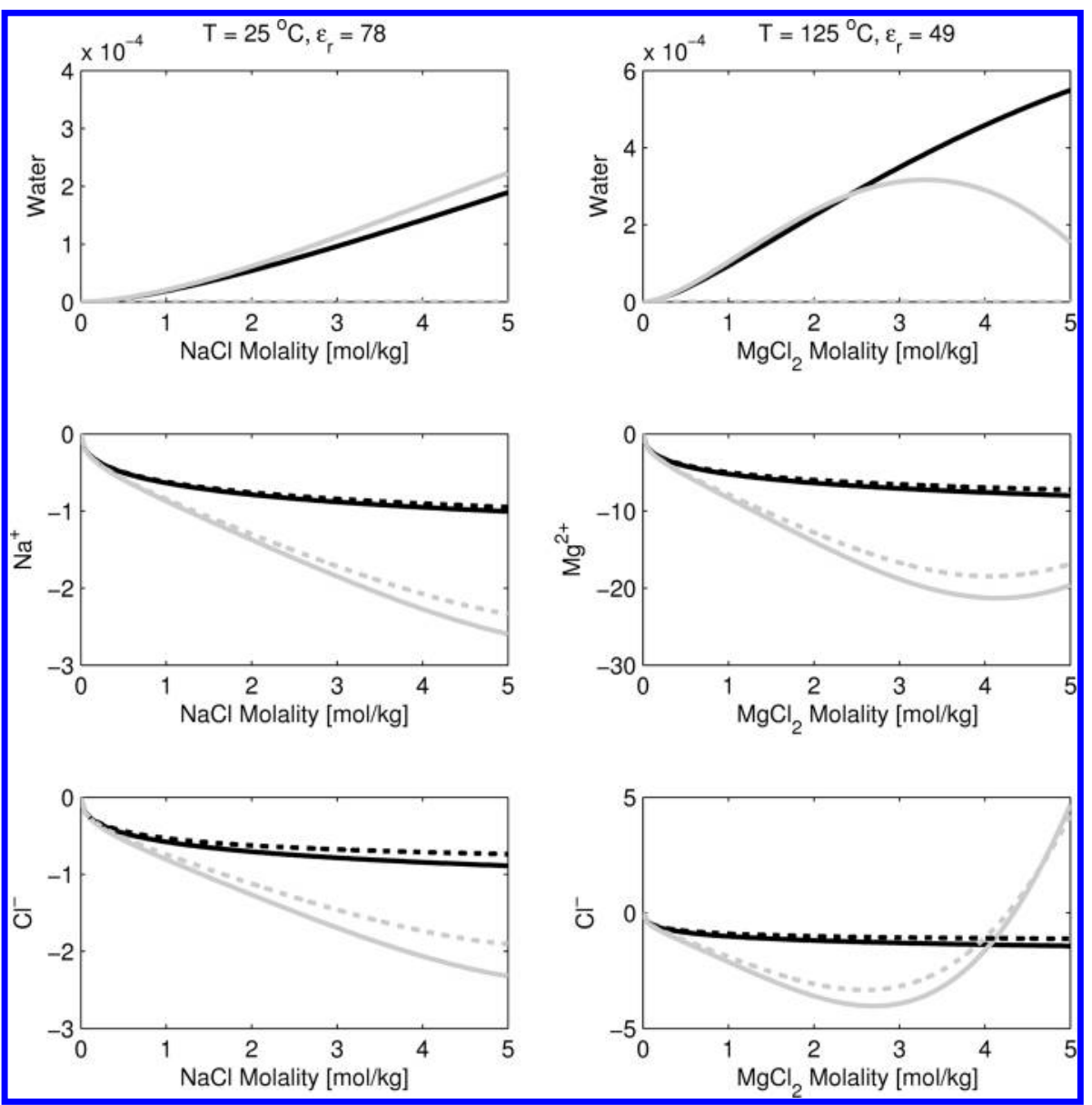

Figure 14. Comparison of the compositional derivative of the Helmholtz energy calculated by MSA (solid) and Debye-Hückel (dashed) at constant temperature using a constant static permittivity (black lines) (shown above the top figure) and the empirical correlation from eq 41 (gray lines). Results are obtained using a constant volume $V=1.3 \mathrm{~L}$.

and Debye-Hückel at constant volume (e.g., same volume in MSA and Debye-Hückel), but as evidenced by Figure 10 and Figure 11, the difference in the volume derivative may lead to a slightly different volume root and thus somewhat different results for the activity coefficients at constant temperature and pressure, depending on the parameters and the other terms in the EoS. Figure 14 shows that the compositional dependence of the static permittivity shown in Figure 3 has a large effect on the compositional derivatives of the Helmholtz energy.

Figure 14 shows that including the compositional dependence of the static permittivity may completely change the behavior of the compositional derivatives at higher concentrations and thus is important to the calculation of activity coefficients. While Figure 11 showed that we have to be careful about the model which we used for the static permittivity, Figure 14 shows that it is of great importance to include the compositional dependence of the static permittivity. The importance of the relative static permittivity on the compositional derivatives was also noted by other authors. ${ }^{5,7,18,22,33,34}$ As the empirical model of the static permittivity does not depend on the concentration of uncharged molecules, only the MSA model provides a small contribution to the compositional derivative of the Helmholtz energy for water, but this contribution is very small compared to the contribution of the ions. If the effect of increasing solvent concentration was included in the model for the static permittivity, both models would yield a larger contribution to the compositional derivative of the Helmholtz energy for water.

\section{CONCLUSION}

The long-range Coulombic forces may be described by either the Debye-Huickel or the MSA theories. The MSA model was derived on the basis of statistical mechanics and includes the effect of an excluded volume on the electrostatic interactions. The results with the two theories were compared numerically in terms of the screening length, the contribution to the residual Helmholtz energy, and the first-order derivatives. Both models predict similar trends with regards to temperature, volume, and compositional dependence of the Helmholtz energy, while only MSA correctly predicts an increase in the screening length when the ion diameter is increased. Nearly identical quantitative results of the two theories are obtained if the distance of closest approach $d_{i}$ of the Debye-Hückel theory is taken as $5 / 6$ of the hard sphere diameter used in the MSA model. It is thus concluded that the two theories will perform similarly if the ion diameter is included as a fitted parameter in the parameter estimation. The static permittivity was found to be a key parameter and the effect of temperature, volume, and composition on the static permittivity will affect the predicted behavior of ions in the solution. The current models for the static permittivity are empirical and this makes it difficult to obtain trustworthy results for multicomponent solutions using the current equations of state for electrolytes. 


\section{ASSOCIATED CONTENT}

\section{S Supporting Information}

Numerical solution procedure for fast and robust solution of the implicit mean spherical approximation equations. This material is available free of charge via the Internet at http:// pubs.acs.org.

\section{AUTHOR INFORMATION}

\section{Corresponding Author}

*E-mail: bmm@kt.dtu.dk. Tel.: +45 45252869. Fax: +45 45882258.

Notes

The authors declare no competing financial interest.

\section{ACKNOWLEDGMENTS}

The authors thank the Department of Chemical and Biochemical Engineering, DTU, and the CHIGP Consortium (Statoil, Mærsk Oil \& Gas, DONG Energy, BP, GASSCO) for funding this research.

\section{REFERENCES}

(1) Kontogeorgis, G. M.; Folas, G. K. Thermodynamic Models for Industrial Applications: From Classical and Advanced Mixing Rules to Association Theories; Wiley: New York, 2010; ISBN: 978-0-470-69726-9.

(2) Thomsen, K; Rasmussen, P. Modeling of vapor-liquid-solid equilibrium in gas-aqueous electrolyte systems. Chem. Eng. Sci. 1999, 54, 1787.

(3) Chen, C. C.; Evans, L. B. A local composition model for the excess Gibbs energy of aqueous electrolyte systems. AIChE J. 1986, 32, 444.

(4) Fürst, W; Renon, H. Representation of excess properties of electrolyte solutions using a new equation of state. AIChE J. 1993, 39 (2), 335.

(5) Myers, J. A.; Sandler, S. I.; Wood, R. H. An equation of state for electrolyte solutions covering wide ranges of temperature, pressure, and composition. Ind. Eng. Chem. Res. 2002, 41, 3282-3297.

(6) Lin, Y.; Thomsen, K.; de Hemptinne, J.-C. Multicomponent equations of state for electrolytes. AIChE J. 2007, 53 (4), 989.

(7) Inchekel, R.; de Hemptinne, J-C; Fürst, W The simultaneous representation of dielectric constant, volume and activity coefficients using an electrolyte equation of state. Fluid Phase Equilib. 2008, 271 $(1-2), 19-27$.

(8) Galindo, A.; Gil-Villegas, A.; Jackson, G.; Burgess, A. N. SAFTVRE: Phase behavior of electrolyte solutions with the statistical associating fluid theory for potentials of variable range. J. Phys. Chem. B 1999, 103, 10272.

(9) Cameretti, L. F.; Sadowski, G.; Mollerup, J. M. Modeling of aqueous electrolyte solutions with perturbed-chain statistical associated fluid theory. Ind. Eng. Chem. Res. 2005, 44, 3355-3362.

(10) Tan, S. P.; Adidharma, H.; Radosz, M. Statistical associating fluid theory coupled with restricted primitive model to represent aqueous strong electrolytes. Ind. Eng. Chem. Res. 2005, No. 44, 4442445 .

(11) Zhao, H.; dos Ramos, M. C.; McCabe, C. Development of an equation of state for electrolyte solutions by combining the statistical associating fluid theory and the mean spherical approximation for the nonprimitive model. J. Chem. Phys. 2007, 126, 244503-17.

(12) Debye, P.; Hückel, E. Zur theorie der elektrolyte. Phys. Z. 1923, 24, 179-207.

(13) Blum, L. Mean spherical model for asymmetric electrolytes. Mol. Phys. 1975, 30 (5), 1529-1535.

(14) Blum, L. In Theoretical Chemistry: Advances and Perspectives; Academic Press Inc, 1980; Vol. 5, pp 1-65, ISBN: 9780126819052.

(15) Soave, G Equilibrium constants from a modified RedlichKwong equation of state. Chem. Eng. Sci. 1972, 1197.
(16) Chapman, W. G.; Gubbins, K. E.; Jackson, G.; Radosz, M. New reference equation of state for associating liquids. Ind. Eng. Chem. Res. 1990, 29, 1709.

(17) Born, M. Volumen und Hydrationswärme der Ionen. Z. Phys. 1920, 1 (1), 45.

(18) Raatschen, W.; Harvey, A. H.; Prausnitz, J. M. Equation of state for solutions of electrolytes in mixed solvents. Fluid Phase Equilib. 1987, 38, 19-38.

(19) Paricaud, P.; Galindo, A.; Jackson, G. Recent advances in the use of the SAFT approach in describing electrolytes, interfaces, liquid crystals and polymers. Fluid Phase Equilib. 2002, 194-197, 87-96.

(20) Patel, B. H.; Paricaud, P.; Galindo, A.; Maitland, G. C. Prediction of the salting-out effect of strong electrolytes on water + alkane solutions. Ind. Eng. Chem. Res. 2003, 42 (16), 3809-3823.

(21) McQuarrie, D. Statistical Mechanics; University Science Books: Sausalito, CA, 2000; ISBN: 1-891389-15-7.

(22) Michelsen, M. L.; Mollerup, J. M. Thermodynamic Models: Fundamentals \& Computational Aspects; Tie-Line Publications: Holte, Denmark, 2007; ISBN 87-989961-3-4.

(23) Attard, P. Asymptotic analysis of primitive model electrolytes and the electrical double layer. Phys. Rev. E 1993, 48 (5), 3604-3618.

(24) Loehe, J. R.; Donohue, M. D. Recent advances in modeling thermodynamic properties of aqueous strong electrolyte systems. AIChE J. 1997, 43 (1), 180-195.

(25) Onsager, L. Theories of concentrated electrolytes. Chem. Rev. 1933, 18, 73 .

(26) Onsager, L. A Correction to the Poisson-Boltzmann equation for unsymmetrical electrolytes. J. Am. Chem. Soc. 1964, 86 (17), 34213423.

(27) Pitzer, K. S. Thermodynamics of electrolytes, theoretical basis and general equations, J. Phys. Chem., 1973, 77 (2)

(28) Pitzer, K. S. Thermodynamics, 3rd ed.; McGraw-Hill: New York, 1995; ISBN 0-07050221-8.

(29) Høye, J. S.; Stell, G. Thermodynamics of the MSA for simple fluids. J. Chem. Phys. 1977, 67, 439.

(30) Giaquinta, P. V.; Parrinello, M.; Tosi, M. P. Hydrodynamic correlation functions for molten salts. Phys. Chem. Liq. 1976, 5, 305.

(31) Vieillefosse, P. Fluctuations de charge et de masse dans un sel fondu binaire a la limite hydrodynamique. J. Phys. Lett. (Paris) 1977, 38, L43.

(32) Marcus, Y. Ionic radii in aqueous solutions. Chem. Rev. 1988, 88, $1475-1498$.

(33) Wu, J.; Prausnitz, J. M. Ind. Eng. Chem. Res. 1998, 37, 16341643.

(34) Simon, H.; Kistenmacher, H.; Prausnitz, J. M.; Vortmeyer, D. Chem. Eng. Process. 1991, 29, 139-146.

(35) Springer Materials, The Landolt Börnstein Database; 2011, http:// www.springermaterials.com (September 1, 2011).

(36) Akhadov, Y. Y. Dielectric Properties of Binary Solutions: A Data Handbook; Elsevier: New York, 1980; ISBN 978-0080236001.

(37) Barthel, J.; Buchner, R.; Münsterer, M. Electrolyte Data Collection, Part 2: Dielectric Properties of Water and Aqueous Electrolyte Solutions; Dechema: Frankfurt, Germany, 1995; ISBN 3-926959-62-2.

\section{NOTE ADDED AFTER ASAP PUBLICATION}

After this paper was published online March 30, 2012, several minor corrections were made to the text. The corrected version was reposted April 3, 2012. 